\title{
¿Cómo prevenir las agresiones a los médicos?
}

\author{
How to prevent attacks on doctors?
}

\section{Carlos Enrique Alvarez Agudelo ${ }^{1}$}

${ }^{1}$ CS FOIETES- Benidorm, Alicante, España

\section{Resumen}

Este trabajo es un comentario del artículo: Raveel A, Schoenmakers B. Interventions to prevent aggression against doctors: a systematic review. BMJ Open. 2019;9:e028465. doi: 10.1136/ bmjopen-2018-028465.

\section{Abstract}

This text is a commentary on the article: Raveel A, Schoenmakers B. Interventions to prevent aggression against doctors: a systematic review. BMJ Open. 2019;9:e028465. doi: 10.1136/ bmjopen-2018-028465.

Sección coordinada por

Consol Serra (consol.serra@upf.edu) | Mª del Mar Seguí (mm.segui@ua.es)

Fechas · Dates

Recibido: 2019.11 .22

Publicado: 2020.01.15
Correspondencia $\cdot$ Corresponding Author

Carlos Enrique Alvarez Agudelo

cealvareza@gmail.com

CS FOIETES- Benidorm, Alicante, España 


\section{Resumen del artículo comentado(1)}

Objetivo: Analizar la evidencia en intervenciones para prevenir agresiones a médicos.

Diseño: Revisión sistemática de la literatura y valoración utilizando instrumentos y guías estandarizados.

Fuentes de datos: Pubmed, Embase, Turning Research into Practice (TRIP), Cochrane y Psycharticle, Google Académico y www.guideline.gov.

Criterios de selección: Se buscaron resúmenes publicados en inglés entre enero de 2000 y enero 2018. Se eligieron estudios enfocados a la prevención y factores de riesgo de violencia tipo II en centros de atención primaria, departamentos de psiquiatría y departamentos de urgencias hospitalarias.

Extracción de los datos y síntesis: Se seleccionaron estudios de intervención, agrupados en estudios cuantitativos y cualitativos. Las revisiones sistemáticas se informaron independientemente. Para cada estudio, diseño y tipo de intervención se analizaron los aspectos más relevantes. La calidad de la información se basó en el método GRADE y el GRADE-Confianza en la Evidencia obtenida en las Revisiones de Investigación Cualitativas (CERQUAL).

Resultados: Se incluyeron 44 estudios. Un ensayo clínico doble ciego aportó evidencia moderada de la disminución de la violencia mediante un programa de prevención. Los principales factores de riesgo fueron los tiempos de espera prolongados, la discrepancia entre las expectativas de los pacientes y los servicios recibidos, el abuso de sustancias y la presencia de enfermedades psiquiátricas. Se observó que una adecuada distribución arquitectónica, iluminación, rutas de evacuación, barreras de protección para recepcionistas, cámaras de vigilancia, alarmas, botones de pánico y además el adecuado desarrollo planes preventivos en el lugar de trabajo son intervenciones que pueden recomendarse para reducir los factores de riesgo, aunque no existe una buena evidencia sobre su efectividad. Un ensayo clínico aleatorizado aportó evidencia de que la valoración del riesgo de violencia del paciente, combinada con acciones preventivas adecuadas pueden disminuir de manera significativa las agresiones más violentas en unidades de psiquiatría. La aplicación de técnicas de des-escalamiento del conflicto durante episodios de agresión son muy recomendados. La realización de un informe posterior al incidente seguido de un análisis causal del mismo aporta las bases para una mejora y optimización de los protocolos de prevención de agresiones.

Conclusiones: Esta revisión documenta intervenciones para prevenir y disminuir agresiones contra médicos. Las agresiones a los médicos son un serio peligro laboral. Existe evidencia moderada que los programas integrales de prevención de la violencia disminuyen el riesgo de violencia entre pacientes y profesionales. Esta revisión no obtuvo datos numéricos suficientes para realizar un metaanálisis. Un estudio de cohortes a gran escala podría mejorar el conocimiento sobre la efectividad de estas intervenciones. 


\section{Comentario del artículo(1)}

Las agresiones a médicos y otros trabajadores sanitarios es una causa muy importante de riesgo laboral en este colectivo. Las agresiones pueden ser de tipo verbal, físico o psicológico con serias implicaciones en la relación con los pacientes, sus familias y los mismos profesionales.

Se trata de un fenómeno en aumento, aunque existe todavía una desproporción entre el número de incidentes reportados y los reales, y tiene más influencia en algunos ámbitos de mayor riesgo como son los servicios de urgencias, psiquiatría y de atención primaria. La prevalencia de las agresiones al personal sanitario, y de acuerdo con diferentes estudios, oscila entre el $36 \%$ y el $70 \%$ aproximadamente; el $55 \%$ se corresponde a agresiones verbales y el $23 \%$ a agresiones físicas, y algunos autores han reportado que hasta el $91 \%$ de los médicos de familia han sufrido algún tipo de agresión durante su carrera profesional. La incidencia por casos de violencia asociados a consumo de drogas o alcohol es del $44 \%$ en servicios de urgencias y a nivel intrahospitalario los eventos por violencia a sanitarios oscilan entre el 8 y $15 \%$ al año(2,3,4).

En la mayoría de los casos el agresor es el propio paciente o un familiar de este, y pueden tener como consecuencia daños físicos o psicológicos, y repercutir en la disminución de la satisfacción profesional y desafección que pueden afectar la calidad del cuidado. Si bien el impacto de las agresiones en el trabajo está bien documentado, no hay evidencia de cómo prevenir o intervenir ante una situación peligrosa, ni tampoco hay consenso sobre planes de seguridad recomendados. En la revisión se han agrupado las intervenciones en:

\section{Medidas y programas preventivos pre-incidente}

Están dirigidas a disminuir el impacto de la violencia en el trabajo, donde se analiza la evaluación de riesgos del lugar de trabajo, prevención de riesgos, entrenamiento y educación en seguridad. Algunos programas incluyen el modelo de hacer y verificar como medida de mejora continua, incluyendo registros y evaluación ${ }^{(5)}$. La utilidad de este tipo de intervenciones se ha confirmado con una evidencia moderada en la reducción del riesgo de agresión, aunque no se demostró una disminución en el número de agresiones físicas. Sin embargo, los resultados pueden estar influidos por el subregistro de eventos.

En general los programas analizados no demostraron una disminución de los eventos violentos. Probablemente, la gran variedad de características en los servicios incluidos hace que un programa genérico no se aplique de igual manera en cada área estudiada, lo cual se contrasta con otros estudios en los que, por ejemplo, en una unidad psiquiátrica italiana se realizaron cambios en la estructura física logrando una reducción significativa del número de agresiones por profesional.

Entre los factores de riesgo de agresión reconocidos están los tiempos de espera prolongados, la discrepancia profesional-paciente en cuanto a los servicios ofre- 
cidos y sus expectativas, o la presencia de abuso de alcohol o drogas y de enfermedades psiquiátricas.

Los cambios en la infraestructura incluyen mejoras en la iluminación interna y externa, mayor espacio de salas de espera, salidas y rutas de escape, cámaras de vigilancia, botones anti-pánico, alarmas portátiles, inclusive detectores de metales como en algunos centros de urgencias de USA, aunque no se ha estudiado su utilidad(6).

Se recomienda tomar medidas de seguridad en los centros de urgencias nocturnas que atienden domicilios, como centralizar esta atención y dotar de acompañantes de seguridad para este fin, además de otras recomendaciones que pueden tener utilidad para evitar agresiones.

El entrenamiento del personal en habilidades de comunicación y en disminución del conflicto deben ser incluidas como parte del programa de prevención ${ }^{(4,7)}$.

\section{Intervenciones durante el incidente}

Existen guías y manuales sobre las técnicas de comunicación de reducción del conflicto, consistentes en transmitir tranquilidad verbal, una relación colaborativa, un estado de calma, siguiendo un ciclo de escucha activa y respuesta asertiva hasta que se logre que el paciente entre en razón, que escuche y actúe acorde a la situación. Este ciclo puede repetirse varias veces hasta lograr neutralizar el conflicto. Cuando estas técnicas no dan el resultado esperado, debe asegurarse la seguridad personal desalojando el lugar y/o activando el protocolo de emergencia. Las medidas de intervención restrictiva solo deben aplicarse de acuerdo con protocolos que preserven los derechos humanos y solo deben ser utilizados en situaciones extremas y en ambientes determinados como son los servicios de urgencias o de psiquiatría.

\section{Medidas post-incidentes}

Es muy importante evitar la trivialización de los incidentes de violencia y la cultura del silencio. El no reportar los incidentes genera un aumento no solo de la frecuencia sino de la severidad de los nuevos episodios de agresión. De esta manera se busca evitar que se normalice la violencia como parte del trabajo, y de igual manera la estigmatización de los agredidos de no ser juzgados por incompetencia. El apoyo de compañeros de trabajo y responsables es fundamental, así como la notificación de los incidentes, su valoración y el análisis, buscando una respuesta en grupo que potencie las soluciones, disminuya la normalización de la violencia y la adecuada aplicación de políticas contra las agresiones. También las organizaciones deben suministrar y organizar el apoyo y asistencia a los profesionales agredidos y prevenir las consecuencias a corto, medio y largo plazo.

En esta revisión bibliográfica los estudios incluidos aportan evidencia moderada sobre la prevención de la violencia laboral tipo II a sanitarios (siempre existe relación profesional entre el agresor y la víctima), y sus factores de riesgo aplicados 
a servicios de psiquiatría, urgencias hospitalarias y de atención primaria, donde existe mayor conflicto. La mayoría de los estudios incluidos provienen de países con mayor desarrollo donde es frecuente esta problemática. Sin embargo, no se menciona ningún de países con bajos recursos, donde esta problemática podría ser mayor por el riesgo de conflicto secundario a la situación social o económica, limitando de esta manera la generalización de los resultados. La revisión incluye estudios que aportan una buena descripción de las diferentes acciones recomendadas en cada estadio. Sin embargo, la mayoría de las recomendaciones y acciones propuestas tienen una evidencia limitada por la baja calidad metodológica y falta de estudios.

Si bien una de las medidas más efectivas es la utilización de técnicas de comunicación adecuadas que permitan el des-escalamiento del conflicto, es un recurso que no es fácil aplicar, que no todos los profesionales tienen disponible y sobre todo que se limita a procesos iniciales de violencia verbal(8).

Son necesarios estudios prospectivos y aleatorizados, basados en las recomendaciones de la OMS y la $\mathrm{OIT}^{(9)}$, que permitan establecer la efectividad de las diversas estrategias para afrontar la violencia en el trabajo en centros sanitarios, para protocolizarlas y sistematizarlas, aplicables a todos los niveles de atención a pacientes, que puedan prevenir efectivamente las agresiones a los sanitarios.

\section{Referencias}

1. Raveel A, Schoenmakers B. Interventions to prevent aggression against doctors: a systematic review. BMJ Open. 2019;9:e028465. doi: 10.1136/ bmjopen-2018-028465

2. Hills DJ, Joyce CM, Humphreys JS. A national study of workplace aggression in Australian clinical medical practice. Med J Aust. 2012;197:336-40.

3. Vorderwülbecke, F; Feistle, M; Mehring, M; Schneider, A; Linde, K. Aggression and violence against primary care physicians-a nationwide questionnaire survey. Dtsch Arztebl Int. 2015;112:159-65.

4. Judith Arnetz; Lydia Hamblin; Jim Russell; Mark Upfal; Mark Luborsky; James Janisse; Lynnette Essenmacher; Preventing Patient-to- Worker violence in hospitals: outcome of a randomized controlled intervention. J Occup Environ Med. 2017;59:18-27.

5. Joanna Briggs Institute. Checklist for Analytical Cross Sectional Studies. 2017 [Citado 15 Oct 2019]. Disponible en: https://joannabriggs.org/sites/default/files/2019-06/JBI_Critical_Appraisal-Checklist_for_Analytical_Cross_Sectional_Studies2017.docx.

6. Serrano Vicente MI, Fernández Rodrigo MT, Satústegui Dordá PJ, Urcola Pardo F. Agresiones a profesionales del sector sanitario en España, revisión sistemática. Rev. Esp Salud Publica. 2019;93:2 de octubre e201910097. 
7. Altemir M, Arteaga A. Protocolo de actuación para prevenir y afrontar agresiones al personal sanitario. Enfermería Clínica. 2018;28,2:125-32.

8. Evans G. OSHA Seeks Comment on Violence Prevention Regulation. Hosp Employee Health. 2017;36,2.

9. International Labour Office/International Council of Nurses/ World Health Organization/Public Services International. Framework Guidelines for Addressing Workplace Violence in the Health Sector. Geneva, International Labour Office, 2002 ISBN 92-2-113446-6. 